Check for updates

Montreal

Cite this as: BMJ 2021;375:n2855 http://dx.doi.org/10.1136/bmi.n2855 Published: 19 November 2021

\section{Covid-19: Doctors will refuse to limit use of antiviral drug to unvaccinated patients, say ethicists}

\author{
Owen Dyer
}

Leading US ethicists have warned that doctors will revolt if the drug regulator authorises an antiviral treatment for covid-19 for use only in people who have not been vaccinated.

Pfizer has applied to the US Food and Drug Administration for emergency authorisation of its new antiviral PF-07321332 (to be marketed as Paxlovid), after promising early trial results indicated that it could cut hospital admissions by $89 \%$ among recently infected adults at high risk of severe illness who were unvaccinated. But the application for the treatment of unvaccinated people only could undermine the US immunisation effort, medical ethicists have warned, by rewarding people who ignored public health advice and penalising those who heeded it.

The final FDA authorisation may not reflect the application, Pfizer has noted, and could yet cover vaccinated people once trial data on that population are submitted.

Arthur Caplan, a professor of bioethics at New York University, told The BMJ that refusing a medicine to a vaccinated person with a breakthrough infection while giving it to a vaccine refuser in the next room was impossible to justify. "No healthcare provider will comply," he said. "The goal of pandemic management is prevention, to prevent transmission. Any step that weakens efforts at prevention that lacks a scientific base is unethical in that it risks undermining support for prevention."

Pfizer spokeswoman Jerica Pitts said that the company submitted data to the FDA as it became available and that it hoped to submit data from trials in two lower risk groups, containing many vaccinated participants, by the end of the year.

The trial of Paxlovid trial in unvaccinated patients was stopped early because of strong results. But Pfizer also began that trial earlier, in July, and did not begin the trial in low risk people until early September.

\section{Off-label use}

James Adams, a medical ethicist and professor of emergency medicine at Northwestern University, Evanston, Illinois, said that Pfizer "cannot really ask for any broader approval" until it had collected evidence of benefit in vaccinated people. But many doctors will not comply with a restriction on prescribing to the vaccinated, he predicted, and will resort to off-label prescribing.

"Speaking as a practising physician, I would look at the safety data and would consider prescribing the medication to vaccinated people who also might be at high risk. This will become very interesting. The government is bulk purchasing so might exercise control," he said. "I suspect that off-label prescribing will be strongly discouraged, but that will not stop many doctors from loudly expressing opinions and, in some cases, perhaps even gaming the system to get access for high risk but vaccinated individuals."

He added, "This new drug might give comfort and confidence to those who wish to remain unvaccinated, but there will remain extraordinary medical, regulatory, and employer pressures for vaccination."

Paxlovid could be authorised for unvaccinated people in a matter of weeks, but the company expects to manufacture only enough to treat 180 ooo people by the end of this year. Production should ramp up swiftly thereafter, with 21 million courses available by next June.

Another new covid antiviral with promising results, Merck's molnupiravir (Lagevrio), was studied in "at risk" people without differentiation on the basis of vaccination status, and its FDA application, submitted in October, is for use in all adults at risk. The UK authorised molnupiravir on 4 November for use in adults with a positive covid test who have at least one risk factor for developing severe illness. ${ }^{1}$

Asked for comment, an FDA spokeswoman replied that, to protect commercial confidentiality and the integrity of the review process, the agency "generally cannot disclose information about unapproved products."

She added, "We are committed to quickly and thoroughly reviewing all submitted applications, including requests for emergency use authorisation, to speed patient access to medicines to prevent or treat covid-19 provided they meet the agency's rigorous standards."

\section{Global access}

Many questions about both antivirals remain unanswered, because their trial results have been issued only in press releases. ${ }^{23}$ Safety concerns include molnupiravir's potential to affect human genes (its trial excluded women who were pregnant or likely to conceive) and the tendency of ritonavir, which must be given with Paxlovid, to interact with other drugs. 4

But if their early results are confirmed the new drugs, as the first oral covid antivirals that can be taken at home, could have a huge effect on the pandemic's death toll, especially in countries with poor vaccine coverage. The US price of both is expected to be in the range of $\$ 700$ ( $€ 520 ; € 620$ ) for a five day therapeutic course, about one third of the cost of monoclonal antibody treatments, which must be given by infusion in a healthcare setting. 
Pfizer and Merck have both signed agreements with the United Nations backed Medicines Patent Pool to license their drugs to a broad range of manufacturers, and both will set tiered prices to make the antivirals affordable in less wealthy countries.

There are already signs, however, that bulk orders from richer countries are monopolising the early supply, as they did with vaccines. Australia, France, Indonesia, Japan, Malaysia, the Philippines, Singapore, South Korea, Thailand, the UK, and the US have all placed orders for one or both antivirals, although the drugs are not yet authorised in most countries.

1 Mahase E. Covid-19: Molnupiravir reduces risk of hospital admission or death by $50 \%$ in patients at risk, MSD reports. BMJ2021;375:n2422. doi: 10.1136/bmj.n2422 pmid: 34607801

2 Merck and Ridgeback's investigational oral antiviral molnupiravir reduced the risk of hospitalization or death by approximately 50 percent compared to placebo for patients with mild or moderate covid-19 in positive interim analysis of phase 3 study. Merck [press release]. 1 Oct 2021.

https://www.merck.com/news/merck-and-ridgebacks-investigational-oral-antiviral-molnupiravirreduced-the-risk-of-hospitalization-or-death-by-approximately-50-percent-compared-to-placebofor-patients-with-mild-or-moderat.

3 Pfizer's novel covid-19 oral antiviral treatment candidate reduced risk of hospitalization or death by $89 \%$ in interim analysis of phase 2/3 Epic-Hr study. Pfizer [press release]. 5 Nov 2021. https://www.pfizer.com/news/press-release/press-release-detail/pfizers-novel-covid-19-oralantiviral-treatment-candidate.

4 Ledford H. Covid antiviral pills: what scientists still want to know. Nature Nov 2021. https://www.nature.com/articles/d41586-021-03074-5.

This article is made freely available for use in accordance with BMJ's website terms and conditions for the duration of the covid-19 pandemic or until otherwise determined by BMJ. You may use, download and print the article for any lawful, non-commercial purpose (including text and data mining) provided that all copyright notices and trade marks are retained. 